\title{
Ulinastatin enhances autophagy against radiation-induced lung injury in mice
}

\author{
Guoxing Zhang ${ }^{1 \#}$, Yujun Du ${ }^{2 \#}$, Ni Sun ${ }^{1}$, Yu Sun ${ }^{1}$, Liying Zhang ${ }^{1}$, Xiaohua $\mathrm{Li}^{3}$, Xiujiang $\mathrm{Li}^{1}$ \\ ${ }^{1}$ Department of Intensive Care Unit, Jilin Cancer Hospital, Changchun, China; ${ }^{2}$ Department of Kidney, ${ }^{3}$ Department of Infectious Diseases, The \\ First Hospital of Jilin University, Changchun, China \\ Contributions: (I) Conception and design: G Zhang, X Li; (II) Administrative support: X Li; (III) Provision of study materials or patients: Y Du; (IV) \\ Collection and assembly of data: G Zhang, X Li, N Sun; (V) Data analysis and interpretation: G Zhang, L Zhang; (VI) Manuscript writing: All \\ authors; (VII) Final approval of manuscript: All authors. \\ "These authors contributed equally to this work. \\ Correspondence to: Xiujiang Li. Department of Intensive Care Unit, Jilin Cancer Hospital, 1018 Huguang Street, Changchun 130012, China. \\ Email: xiujiangli@yandex.com.
}

Background: To investigate the enhancement of autophagy by ulinastatin for protecting against radiationinduced lung injury (RILI) in mice.

Methods: Forty C57BL/6 mice were equally divided into (I) control (C), (II) irradiation (R), (III) ulinastatin (U), (IV) 3-methyladenine (3-MA) (M), and (V) ulinastatin plus 3-MA (U+M) groups. Three mice in each group were infected with adeno-associated virus (AAV) carrying green fluorescent protein (GFP)-1A/1Blight chain 3 (GFP-LC3) in the lung for the marker of autophagy. All mice in R, U, M and U+M groups were given chest irradiation (1 Gy/min, $12 \mathrm{~min}$ ), following injection with normal saline in $\mathrm{C}$ and $\mathrm{U}$ groups, ulinastatin (500,000 IU/kg.d, i.p., 7 d) in U group, 3-MA (10 mg/kg.d, i.p., 7 d) in M group, and ulinastatin plus 3-MA in $U+M$ group. The effects of ulinastatin on lung injury and autophagy were evaluated by electron microscope (EM), immunohistochemistry, mRNA expression levels of collagen alpha-1 (COL1A1), collagen alpha-2 (COL1A2), $\alpha$-smooth muscle actin ( $\alpha$-SMA) and transforming growth factor $\beta 1$ (TGF- $\beta 1$ ), and protein levels of LC3, $\alpha$-SMA, COL1A2, TGF- $\beta 1$, matrix metalloproteinase-2 (MMP-2) and MMP-9.

Results: EM observation revealed that the radiation caused the injury of type I and II alveolar epithelial cells, which was improved by ulinastatin treatment associated with increased the numbers of autophagosomes. GFP-LC3 signals was significantly enhanced by ulinastatin detected by immune histochemical tests. At transcriptional and/or translational levels, ulinastatin significantly enhanced the expression levels of TGF- $\beta 1$ and LC3 but reduced COL1A1, COL1A2, $\alpha$-SMA, MMP-2 and MMP-9 after radiation-induced RILI.

Conclusions: Ulinastatin reduces RILI by enhancing autophagy, which might be a potential therapeutic drug in the protection against RILI.

Keywords: Ulinastatin; radiation-induced lung injury (RILI); autophagy

Submitted Dec 30, 2019. Accepted for publication Jun 12, 2020.

doi: 10.21037/tcr-19-3018

View this article at: http://dx.doi.org/10.21037/tcr-19-3018

\section{Introduction}

Radiotherapy is used in the treatment of pulmonary tumors, although it causes serious side-effects on normal lung tissue, including exudative inflammation in acute phase, interstitial pneumonia in 6-12 weeks and pulmonary fibrosis $(1,2)$. Thoracic radiotherapy may cause two types of radiation-induced lung injury (RILI), radiation pneumonitis and radiation fibrosis (3-5). The radiationcaused lung injury, especially inflammation in the early period, can be controlled by some adjuvants, include glucocorticoids, non-steroidal anti-inflammatory drugs, 
and $\gamma$-interferon. However, the treatment of pulmonary interstitial fibrosis that develops in the later stage remains unsatisfactory. As a broad-spectrum protease inhibitor, ulinastatin inhibits hydrolase activity and has protective effects on the injury of multiple organs, including ventilator-associated and lipopolysaccharide-induced acute lung injuries (6-8). Pretreatment with high-dose of ulinastatin prevents the radiation-induced pulmonary injury in rats (9) and in patients with non-small cell lung cancer after radiation therapy (10). The ulinastatin treatment reportedly suppresses TGF- $\beta$ expression and lung fibrosis, which reduces the injury of irradiation in mice (8). The cumulating evidence shows that ulinastatin protects against LPS-induced acute lung injury by attenuating TLR4/NF$\kappa \mathrm{B}$ pathway activation (11), reduces the resistance of liver cancer cells to epirubicin by inhibiting autophagy (12), and suppresses endoplasmic reticulum stress and apoptosis in the hippocampus of rats with acute paraquat poisoning (13).

The protective effect of ulinastatin on RILI is rarely reported, and the relative mechanism is still unknown. Therefore, the current study was designed to investigate the protective effect on RILI correlated with autophagy in mice. In autophagy, the autophagosomes fuse with lysosomes, which induces the orderly degradation of cells and recycling of cellular components (14). With a bidirectional regulatory effect on inflammatory reaction, autophagy is equally important in promoting and inhibiting inflammation. Autophagy acts as suppressive effects on the inflammation by degrading inflammatory proteins and reducing the level of inflammatory factors (15). On the other hand, autophagy also activates inflammatory cells by promoting the release of inflammation factors, resulting in acceleration of the process of inflammation reaction (16). In the present study, we have established a RILI model in mice, and then treated with ulinastatin and/or 3-methyladenine (3-MA). We have found that the enhancement of autophagy may be an important mechanism of ulinastatin to improve RILI in mice. We present the following article/case in accordance with the ARRIVE reporting checklist (available at http:// dx.doi.org/10.21037/tcr-19-3018).

\section{Methods}

\section{Ethical statement}

All animal tests were carried out in accordance with the US National Institute of Health (NIH) Guide for the Care and Use of Laboratory and approved by Institutional
Animal Care and Use Committee (IACUC) and the ethics committee of the Jilin University (No. Jlzlyy-20180001).

\section{Animals and grouping and materials}

Forty female C57BL/6 mice at the age of 8 weeks and weight of 18-20 g were purchased from animal department of foundation, the Medical College of Jilin University. All mice were exposed to a 12 -h light-dark cycle with free access to standard rodent chow and water. Mice were randomly divided into five groups (8/each group): (I) C, blank control; (II) R, irradiation; (III) $\mathrm{U}$, ulinastatin; (IV) $M$, 3-methyladenine (3-MA); and (V) $U+M$, ulinastatin plus 3-MA. In each group, 3 mice were infected with adenoassociated virus (AAV) carrying combined with green fluorescent protein (GFP) and microtubule-associated protein 1A/1B-light chain 3 (LC3) (HB AAV2/6-mRFPGFP-LC3) for the autophagy detection. All mice were observed by the pathological and immunohistological tests.

\section{Monitoring autophagy in lung tissues}

To monitor the intensity of autophagy flux, the lung tissues of 3 mice in each group were infected HB AAV2/6-mRFPGFP-LC3 $\left(1.2 \times 10^{12} / \mathrm{vg} / \mathrm{mL}\right)$ (Hanbio Biotechnology Co.). The mice were anesthetized by intraperitoneal (i.p.) injection with pentobarbital $(60 \mathrm{mg} / \mathrm{kg})$, and then placed in the supine position. After sterilized with tincture of iodine, the skin in front of trachea was incised. After blunt dissection of the tissues around the trachea, adenovirus $(50 \mu \mathrm{L})$ was injected into the trachea using micro-syringe. The skin was sutured and then sterilized with alcohol. Three weeks after AAV infection, mice were used to build RILI models for different treatments.

\section{Establishment and treatment of RILI animal models}

Eight mice in $\mathrm{C}$ group, including 3 infected with $\mathrm{HB}$ AAV2/6-mRFP-GFP-LC3, were as the blank control. The rest 32 mice in 4 groups, including 3 infected with HB AAV2/6-mRFP-GFP-LC3 in each group, were anesthetized by i.p. injection with pentobarbital $(60 \mathrm{mg} / \mathrm{kg})$, and then placed in the supine position for radiation. The chest was covered with a $23-\mathrm{mm}$-thick wax block to control the dose of radiation. The head and the abdomen were shielded by lead plates. Mouse was placed $1 \mathrm{~m}$ from the radiation source, and the exposure field was $1 \mathrm{~cm} \times 3 \mathrm{~cm}$. The whole thoracic radiation was 
Table 1 The primer for semi-quantitative RT-PCR

\begin{tabular}{lll}
\hline Gene & Forward & Reverse \\
\hline COL1A1 & GGAGGGCGAGTGCTGTGCTTT & GGGACCAGGAGGACCAGGAAGT \\
COL1A2 & TGGTCTTACTGGGAATTTGCTGC & ACCCTGTGGTCCAACGACTCCTCTC \\
$\alpha$-SMA & GACGCTGAAGTATCCGATAGAACACG & CACCATCTCCAGAGTCCAGCACAAT \\
TGF- $\beta 1$ & AGCGGACTACTATGCTAAAGAGGTCACCC & CCAAGGTAACGCCAGGAATTGTTGCTATA \\
GAPDH & CGACTTCAACAGCAACTCCCACTCTTCC & TGGGTGGTCCAGGGTTTCTTACTCCTT \\
\hline
\end{tabular}

performed with a single dose of $12 \mathrm{~Gy}(1.0 \mathrm{~Gy} / \mathrm{min}$ for $12 \mathrm{~min})$. After radiation, mice in $\mathrm{R}$ group did not do any further treatment. The mice in $\mathrm{U}$ group were treated with ulinastatin $(500,000 \mathrm{IU} / \mathrm{kg} \cdot \mathrm{d}$, i.p., $7 \mathrm{~d})$. The dosage of ulinastatin was determined by the pre-experiments, in which 3 mice/each group were administrated with ulinastatin $(300,000,400,000,500,000$ or $600,000 \mathrm{IU} / \mathrm{kg} \cdot \mathrm{d}$, i.p., $7 \mathrm{~d}$, respectively). The results showed that the dose of 500,000 IU/kg.d, i.p., $7 \mathrm{~d}$ was safe. The mice in $\mathrm{M}$ group were treated with 3-MA $(10 \mathrm{mg} / \mathrm{kg} \cdot \mathrm{d}$, i.p., $7 \mathrm{~d})$. The mice in $U_{+} M$ group were treated with ulinastatin (500,000 IU/kg.d, i.p., 7 d) plus 3-MA (10 mg/kg.d, i.p., $7 \mathrm{~d})$. The mice in $\mathrm{C}$ and $\mathrm{R}$ groups were given the same amount of normal saline equaling to the amount of liquid as the mice in the other groups. One week after radiation, all mice were sacrificed by cervical dislocation after anesthetization. A small part of the left lung tissue was fixed with $1 \%$ glutaraldehyde and cut to ultra thin sections. The cell structure was observed by scanning electron microscope (EM) without any staining. The right lung was frozen at $-20^{\circ} \mathrm{C}$ for later biochemical analysis.

\section{Fluorescence}

The left lung was frozen to make slices for fluorescence analysis to detect GFP-LC3 levels using confocal microscope (Olympus bx-51, Japan). The integral optical density (IOD) was determined across a $400 \times$ field, using Image Pro-Plus 6.0 software to reflect the protein expression. A total of 10 fields in each section were randomly chosen for statistical analysis.

\section{Western blots}

The right lung tissues were homogenized in sodium dodecyl sulfate (SDS) lysis buffer containing protease inhibitors on ice for $5 \mathrm{~min}$. Cell debris was removed by centrifugation at $12,000 \times \mathrm{g}$ at $4{ }^{\circ} \mathrm{C}$ for $5 \mathrm{~min}$. The lysate proteins $(30 \mu \mathrm{g})$ were fractionated by SDS-polyacrylamide gel electrophoresis. The separated proteins were transferred to nitrocellulose membranes and probed with primary antibodies, rabbit anti-LC3B antibody (Abcam, Cambridge, $\mathrm{UK}$ ), rabbit monoclonal antibody to $\alpha$-SMA (Abcam), rabbit anti-Col1A2 antibody (Abcam), rabbit anti-TGF- $\beta 1$ antibody (Abcam), rabbit anti-matrix metalloproteinase-2 (MMP-2) antibody (Abcam) and mouse anti- $\alpha$-tubulin IgM monoclonal antibody (Santa Cruz Biotechnology, CA, USA). The membrane was then incubated with horseradish peroxidase-conjugated anti-rabbit or mouse IgG secondary antibody. Immunoblots were visualized by chemiluminescence using a chemiluminescence kit and the specific bands were recorded on X-ray film. $\alpha$-tubulin protein levels were used as a control to verify equal protein loading.

\section{Semi-quantitative RT-PCR}

The total RNA was extracted from the frozen pulmonary tissue using TRIzol reagent (Invitrogen, Shanghai, China). Oligo (dT18)-primed first-strand cDNA was synthesized using a First-Strand cDNA Synthesis kit (Invitrogen) according to the manufacturer's instructions. The cDNA was amplified by 30 cycles of PCR $\left(30 \mathrm{~s}\right.$ at $94^{\circ} \mathrm{C}, 40 \mathrm{~s}$ at $57^{\circ} \mathrm{C}, 40 \mathrm{~s}$ at $72{ }^{\circ} \mathrm{C}$ and, finally, $5 \mathrm{~min}$ at $72^{\circ} \mathrm{C}$ ), followed by one cycle of denaturation of $4 \mathrm{~min}$ at $94{ }^{\circ} \mathrm{C}$. $\beta$-actin was as an internal control. The primer sequences were described in Table 1. PCR products were visualized by ethidium bromide following electrophoresis on a $1 \%$ agarose gel, and quantitated by densitometry using a dualintensity transilluminator equipped with Gel-Pro Analyzer version 3.1 (Media Cybernetics, Bethesda, MD, USA). The relative level of mRNA was expressed as the ratio of the densitometric value of the band normalized to that of $\beta$-actin band. 


\section{Statistical analysis}

The data were presented as mean \pm SD. An ANOVA test was applied to define a difference among treatment groups, which was followed by a post hoc multiple comparisons test to define specific group differences. Values of $\mathrm{P}<0.05$ were considered significant and $\mathrm{P}<0.01$ very significant.

\section{Results}

\section{Ulinastatin induces autophagosomes and prevents from cell damage induced by RILI}

Our original goal was to investigate the role of autophagy in the protective effects of ulinastatin on RILI. Therefore, 1 week after radiation, the EM 5 groups: C, untreated mice as control; R, mice without any treatment after radiation; $\mathrm{U}$, was used to observed the possibly pathological changes in the lung tissues of mice in mice with ulinastatin treatment after radiation; $M$, mice with 3-MA treatment after radiation; and $U+M$, mice with ulinastatin plus 3-MA treatment after radiation. In the lung tissue of mice in $\mathrm{C}$ group, we observed type I alveolar epithelial cells with complete cell membrane and cytoplasmic organelles (Figure 1, A1-2) and type II alveolar epithelial cells with intact lamellar bodies in the cytoplasm (Figure 1, A3-4). A single radiation in mice in $\mathrm{R}$ group caused the injury of type I (Figure 1, B1-2) and II (Figure 1, B3-4) alveolar epithelial cells with rupture, necrosis, mitochondrial cavitation, nuclear bilayer membrane disappears, cytoplasmic cavitation and endoplasmic reticulum expansion. Some autophagosomes with the bilayer membrane structure were in cells (Figure 1, B5-6). After the radiation, the treatment with ulinastatin in mice in $\mathrm{U}$ group kept the relative integrity of the type I (Figure 1, C1-2) and II (Figure 1, C3-4) alveolar epithelial cell structure and increased the numbers of autophagosomes (Figure 1, C5-6). After the radiation, the treatment with 3-MA, an autophagy inhibitor, in mice in $M$ group caused collapsed type I (Figure 1, D1-2) and II (Figure 1, D3-4) alveolar epithelial cells with mitochondrial cavitation and endoplasmic reticulum expansion. After radiation, the treatment with ulinastatin plus 3-MA in mice in $U+M$ group resulted in type I (Figure 1, E1-2) and II (Figure 1, E3-4) alveolar epithelial cells with the mitochondrial cavitation of and the autophagosome reduction (Figure 1, E5-6). The data suggest that ulinastatin increases autophagy and improves the lung injury induced by radiation.

\section{Ulinastatin increases the level of LC3, the marker of autophagy}

To monitor the intensity of autophagy flux, we infected mouse lung tissues ( $\mathrm{n}=3$ /each group) with HB AAV2/6mRFP-GFP-LC3. Three weeks after the AAV infection, the mice were differently treated in 5 groups. The lung tissues were cut into slides and the possible green fluorescence labelled LC3 (GFP-LC3) as an autophagy marker was detected by the fluorescence confocal microscope. In the lung tissue of untreated mice in $\mathrm{C}$ group, no GFPLC3 were detected (Figure 2A). A single radiation in mice in R group induced GFP-LC3 signals (Figure $2 B$ ). The ulinastatin treatment after radiation in mice in $\mathrm{U}$ group significantly enhanced GFP-LC3 signals (Figure 2C). The 3-MA treatment after radiation in mice in $M$ group decreased GFP-LC3 signals to be almost invisible (Figure 2D). The ulinastatin plus 3-MA treatment after radiation in mice in $U_{+} M$ resulted in alleviated GFPLC3 signals (Figure 2E). The data suggest that ulinastatin increases autophagy in the lung tissue after radiation.

\section{Ulinastatin affects the transcriptional levels of molecules involving autophagy}

COL1A1 and COL1A2 genes encode collagen type I $\alpha 1$ and $\alpha 2$ chains, respectively, which are mostly expressed in fibrogenesis (17). The decrease in the levels of COL1A1 and COL1A2 was observed at parturition in the amnion tissue (18). $\alpha$-smooth muscle actin ( $\alpha$-SMA) is an inconsistent marker of fibroblasts (19), and decreased in autophagy (20). Transforming growth factor $\beta 1$ (TGF- $\beta 1$ ) induces autophagy and plays a major role in the formation of myofibroblasts in renal epithelial cells $(21,22)$. By Semiquantitative RT-PCR, we measured the mRNA expression levels of COL1A1, COL1A2, $\alpha$-SMA and TGF- $\beta 1$ in lung tissues of mice in 5 groups (Figure 3; Table 2). We used ANOVA and hoc post test to compare the differences between groups ( $n=3 /$ each group). The significant differences between groups are labeled in Figure 3. The data demonstrate that (I) ulinastatin inhibits but 3-MA enhances the expression of COL1A1, COL1A2 and $\alpha$-SMA induced by radiation, and (II) ulinastatin enhances but 3-MA reduces the TGF- $\beta 1$ expression induced by radiation, suggesting that the enhancement of autophagy associated with the expression levels of these genes by ulinastatin may be an important mechanism to improve the injury by RILI. 

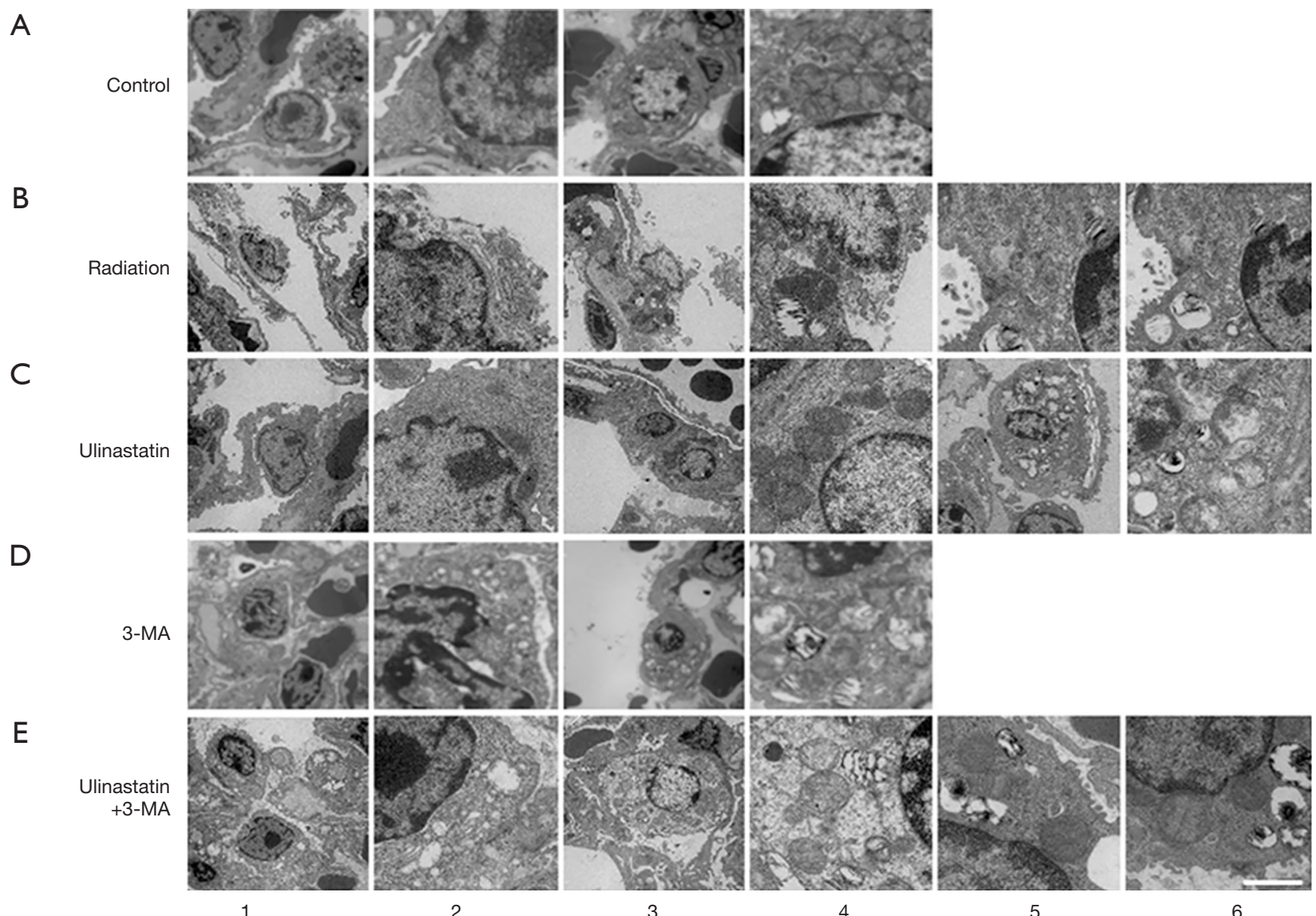

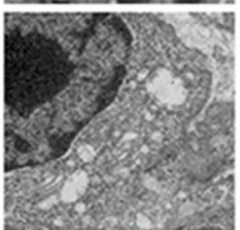

2

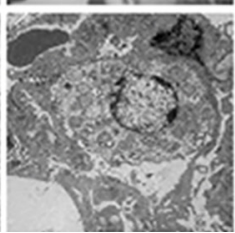

3

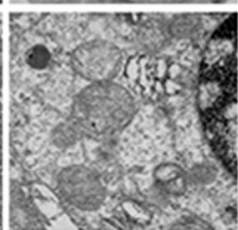

4

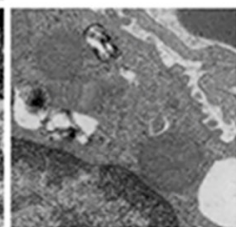

5

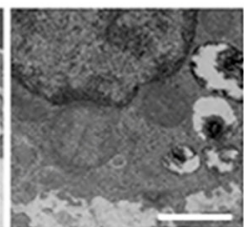

6

Figure 1 Ulinastatin improves cell damage induced by RILI in lung tissues detected by electron microscopy. The lung tissue was fixed with $1 \%$ glutaraldehyde. The cell structure was observed by scanning EM without any staining. (A) The control group: type I (A1-2) and II (A3-4) alveolar epithelial cells with intact cell membrane and cytoplasmic organelles. (B). The radiation group: type I (B1-2) and II (B34) alveolar epithelial cells with rupture, necrosis, mitochondrial cavitation, nuclear bilayer membrane disappears, cytoplasmic cavitation and endoplasmic reticulum expansion, some autophagosomes with the bilayer membrane structure in cells (B5-6); (C) The ulinastatin group: relatively complete type I (C1-2) and II (C3-4) alveolar epithelial cells and more autophagosomes in cells (C5-6); (D) the 3-AM group: the collapsed type I (D1-2) and II (D3-4) alveolar epithelial cells with mitochondria cavitation and endoplasmic reticulum expansion; (E) The ulinastatin plus 3-MA group: type I (E1-2) and II (E3-4) alveolar epithelial cells with mitochondrial cavitation, and some autophagosomes in cells (E5-6). The scale bar $=2.5 \mu \mathrm{m}$.

\section{Ulinastatin regulates the expression levels of proteins associated with autophagy}

As gelatinases, matrix metalloproteinases-2 and -9 (MMP-2 and MMP-9) are important in the pathogenesis of pulmonary diseases (23), and degreased in autophagy (24). Therefore, we measured the protein expression levels of LC3, $\alpha$-SMA, COL1A2, TGF- $\beta 1$, MMP-2 and MMP-9 in lung tissues of mice in 5 groups. The homogenates of lung tissues were subjected to Western blots (n=3/each group) (Figure 4). The protein signals were normalized to the signal of $\alpha$-tubulin and compared as relative protein expression level (fold) by ANOVA and hoc post test (Figure 4; Table 3). The significant differences between groups are labeled in Figure 4. The data demonstrate that (I) ulinastatin inhibits but 3-MA enhances the expression of $\alpha$-SMA, COL1A2, MMP2 and MMP-9 induced by radiation, and (II) enhances but 3 -MA inhibits the expression of LC3 TGF- $\beta 1$, induced by radiation, suggesting that the introduction of autophagy by ulinastatin in radiation-reduced RILI injury is associated with the regulated expression levels of these proteins. 
A
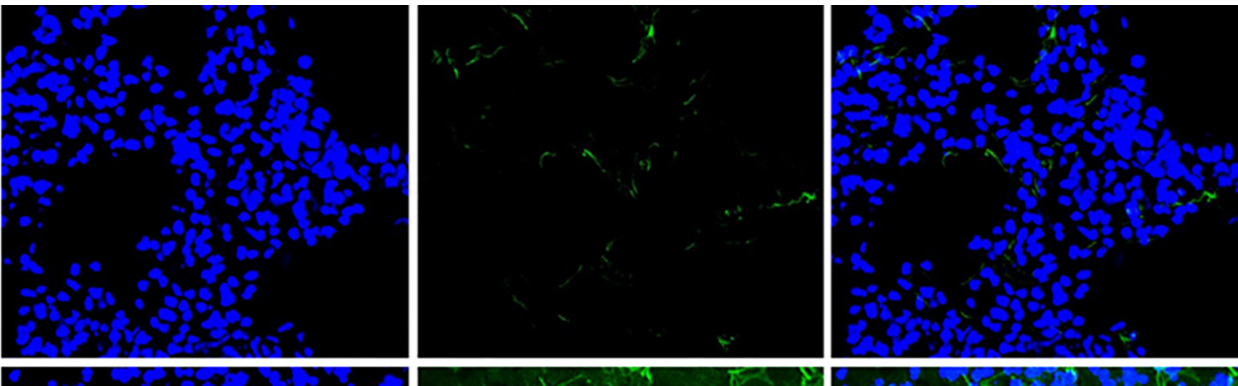

B
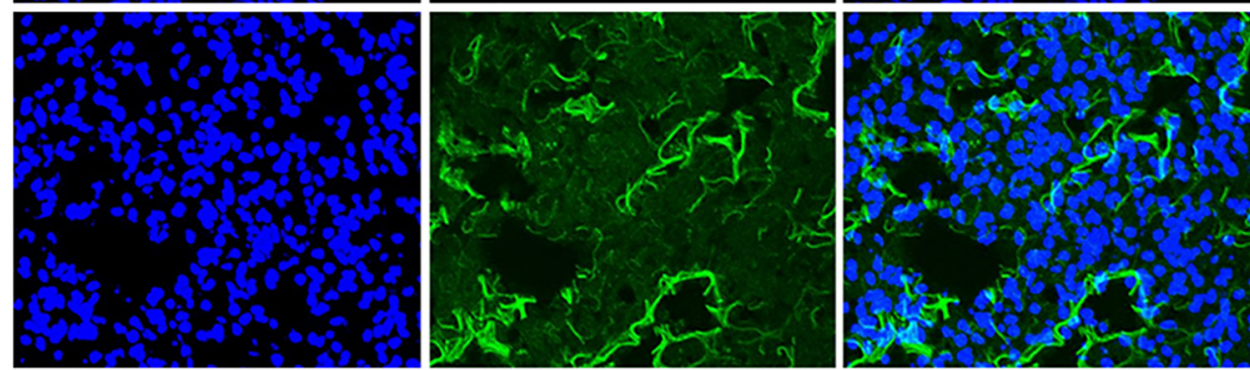

C
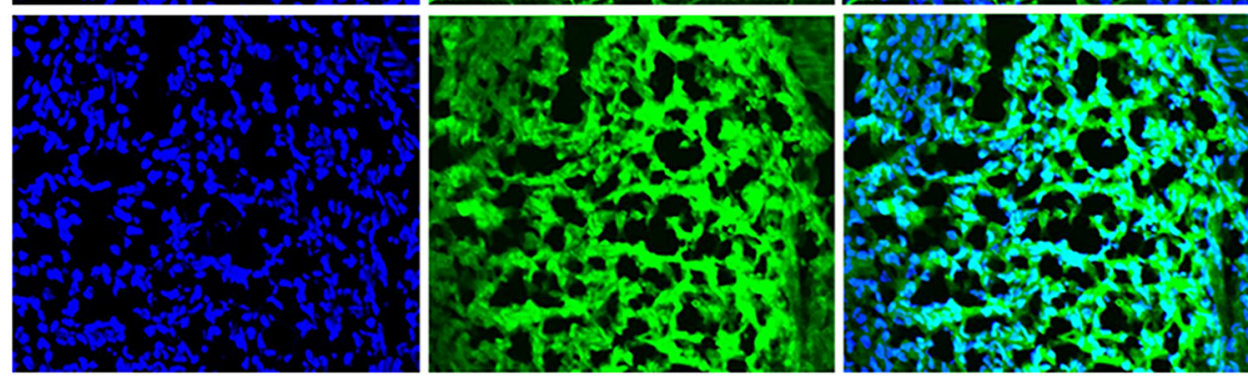

D
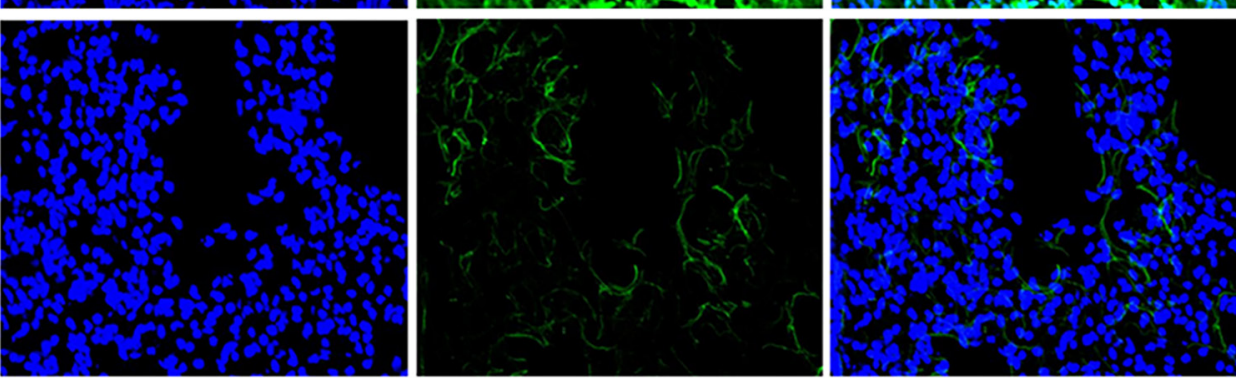

E

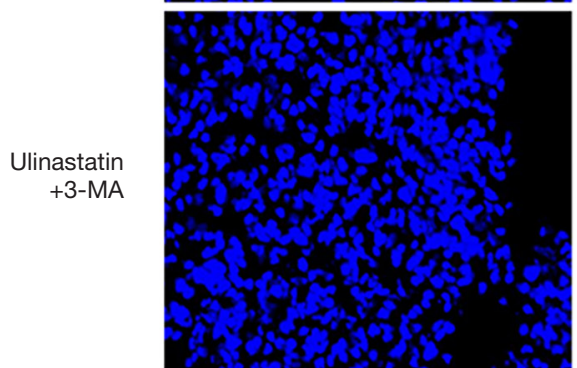

1

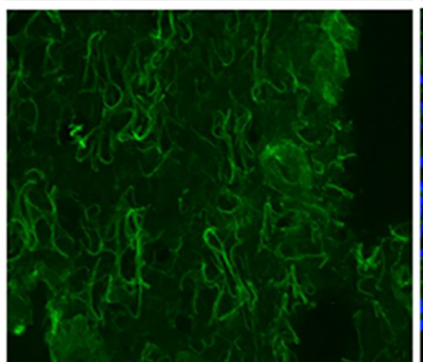

2

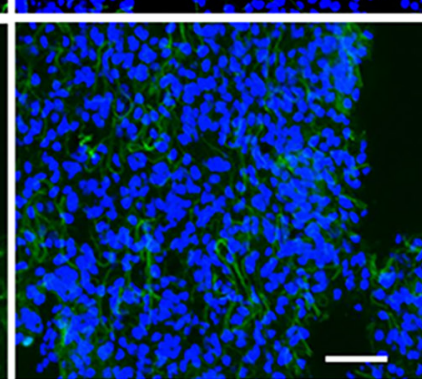

3

Figure 2 Ulinastatin enhances of the GFP-LC3 expression detected by fluorescence confocal microscope. The lung tissues of 3 mice in each group were infected with or without HBAAV2/6-mRFPGFP-LC3. The green fluorescence labelled LC3 (GFP-LC3) in the lung tissue of mice with different treatment were detected under fluorescence microscope without any staining. (A) The control group: no detected GFPLC3 signals; (B) the radiation group: some GFP-LC3 signals; (C) the ulinastatin group: significantly enhanced GFP-LC3 signals; (D) the 3-MA group: almost invisible GFP-LC3 signals. (E) the ulinastatin +3-MA group: alleviated GFP-LC3 signals. Blue: nuclei; green: GFPLC3. The scale bar $=100 \mu \mathrm{m}$. 

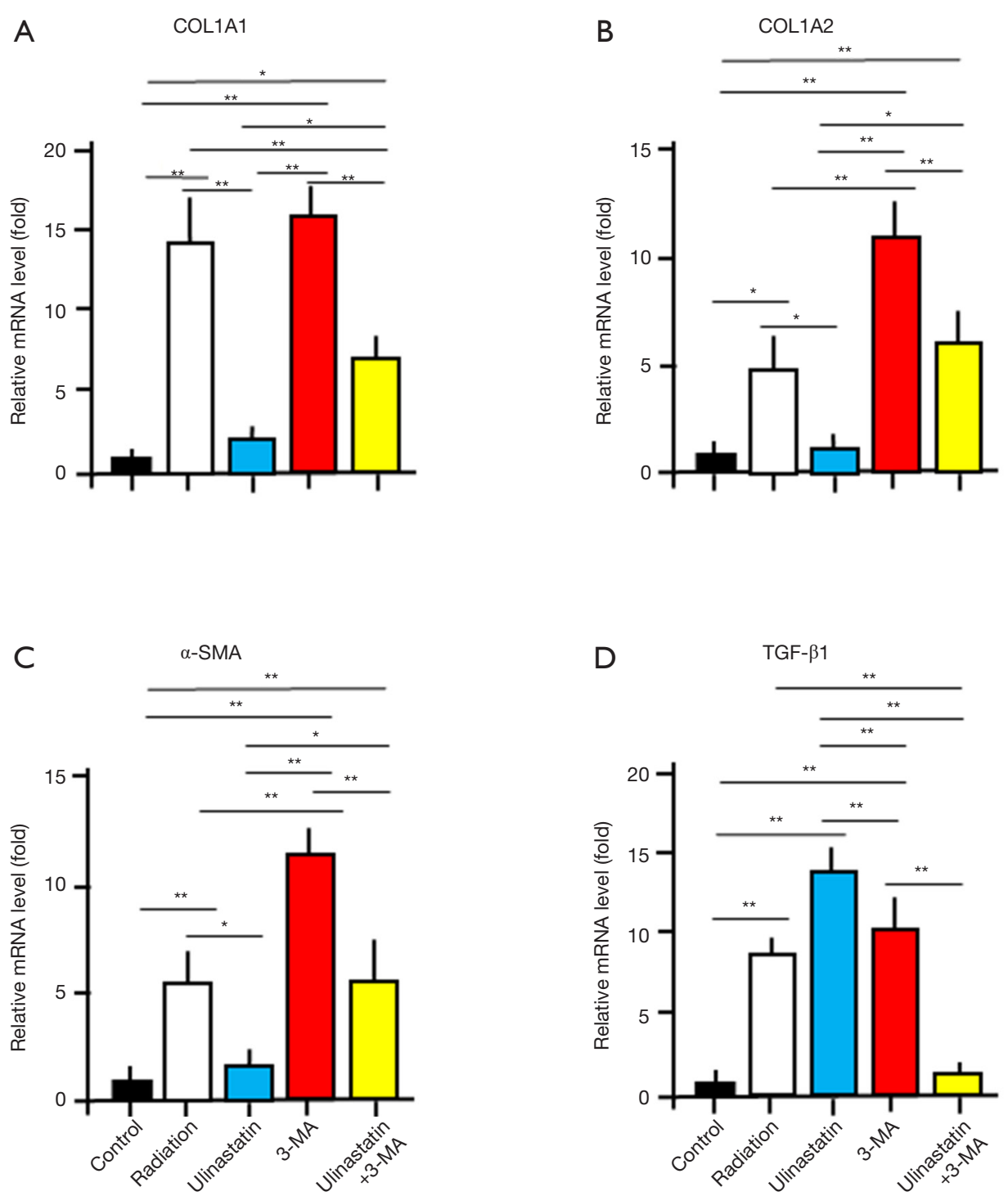

Figure 3 The relative mRNA expression levels of COL1A1 (A), COL1A2 (B), $\alpha$-SMA (C) and TGF- $\beta 1$ (D) in lung tissues of mice in the groups of control $(\mathrm{C})$, radiation $(\mathrm{R})$, ulinastatin $(\mathrm{U}), 3-\mathrm{MA}(\mathrm{M})$ and ulinastatin plus 3-MA (U+M) (n=3/each group). AVONA and hoc post test, *, $\mathrm{P}<0.05 ;{ }^{* *}, \mathrm{P}<0.01$.

Table 2 Relative mRNA levels (fold) in lung tissues of mice with different treatments

\begin{tabular}{lcccr}
\hline Group & COL1A1 & COL1A2 & $\alpha$-SMA & TGF- $\beta 1$ \\
\hline C & $1.00 \pm 0.16$ & $1.00 \pm 0.10$ & $1.00 \pm 0.17$ & $1.00 \pm 0.13$ \\
R & $14.01 \pm 2.81$ & $4.96 \pm 1.43$ & $5.45 \pm 1.03$ & $8.37 \pm 1.12$ \\
U & $2.04 \pm 0.38$ & $1.09 \pm 0.24$ & $1.45 \pm 0.18$ & $15.68 \pm 2.03$ \\
M & $16.09 \pm 1.94$ & $11.08 \pm 1.44$ & $11.34 \pm 1.13$ & $9.95 \pm 2.46$ \\
U+M & $6.94 \pm 1.14$ & $6.05 \pm 1.35$ & $5.56 \pm 1.28$ & $2.45 \pm 0.36$ \\
P & 0.000 & 0.000 & 0.000 & 0.000 \\
\hline
\end{tabular}

Group: C, control; R, irradiation; U, ulinastatin; M, 3-methyladenine (3-MA); U+M, ulinastatin plus 3-MA. P, analyzed by ANOVA. 


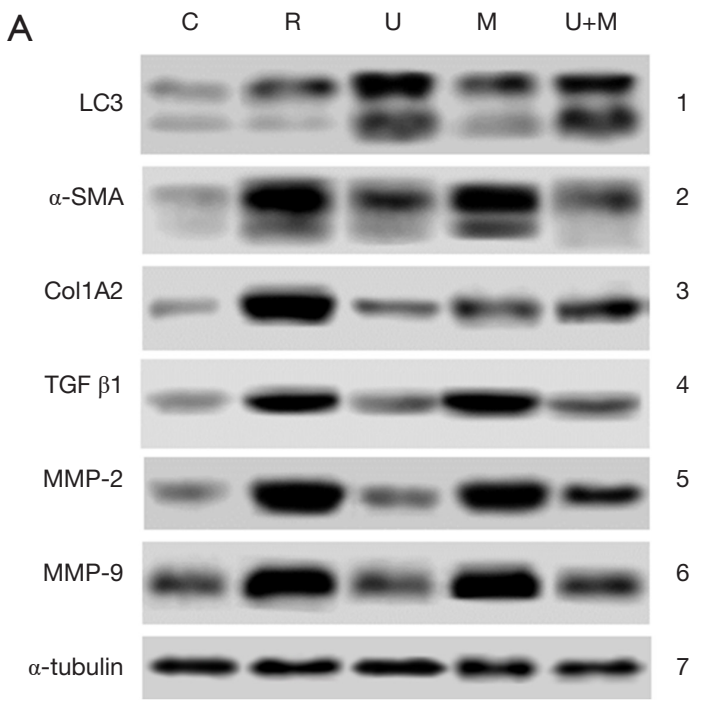

B
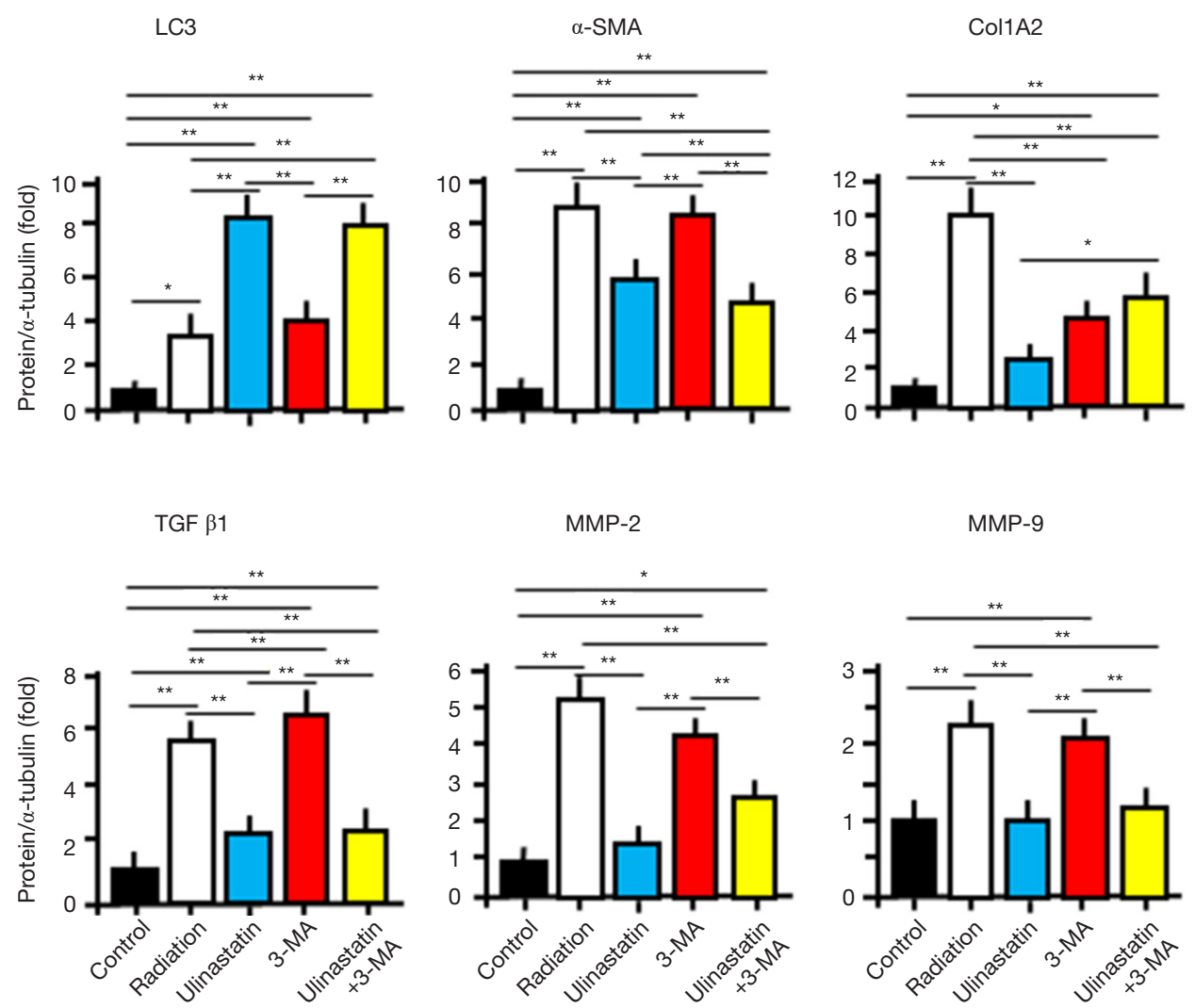

Figure 4 Ulinastatin increases LC3 but reduces $\alpha$-SMA, COL1A2, TGF- $\beta 1$, MMP-2 and MMP-9 induced by radiation. (A) The lung tissue homogenates of mice in the groups of control (C), radiation (R), ulinastatin (U), 3-MA (M) and ulinastatin plus 3-MA (U+M) (n=3/each group) were subjected to Western blots. (B) The protein signals were normalized to the signal of $\alpha$-tubulin, and then compared as relative protein expression level by ANOVA and hoc post test. *, $\mathrm{P}<0.05$; ${ }^{* *}, \mathrm{P}<0.01$. 
Table 3 Relative protein levels (fold) in lung tissues of mice with different treatments

\begin{tabular}{lcccccc}
\hline Group & LC3 & $\alpha$-SMA & COL1A2 & TGF- $\beta 1$ & MMP-2 & MMP-9 \\
\hline C & $1.00 \pm 0.15$ & $1.00 \pm 0.14$ & $1.00 \pm 0.13$ & $1.00 \pm 0.12$ & $1.00 \pm 0.18$ & $1.00 \pm 0.11$ \\
R & $3.01 \pm 0.51$ & $9.23 \pm 1.53$ & $10.17 \pm 1.89$ & $5.56 \pm 0.71$ & $5.17 \pm 0.73$ & $2.27 \pm 0.32$ \\
U & $8.41 \pm 0.84$ & $5.76 \pm 0.76$ & $2.36 \pm 0.31$ & $2.04 \pm 0.29$ & $1.37 \pm 0.18$ & $0.96 \pm 0.12$ \\
M & $3.70 \pm 0.43$ & $8.19 \pm 0.94$ & $4.48 \pm 0.68$ & $6.60 \pm 0.73$ & $4.42 \pm 0.59$ & $2.04 \pm 0.34$ \\
U+M & $8.05 \pm 1.02$ & $4.69 \pm 0.61$ & $5.70 \pm 0.84$ & $2.90 \pm 0.39$ & $2.56 \pm 0.31$ & $0.18 \pm 0.16$ \\
P & 0.000 & 0.000 & 0.000 & 0.000 & 0.000 \\
\hline
\end{tabular}

Group: C, control; R, irradiation; U, ulinastatin; M, 3-methyladenine (3-MA); U+M, ulinastatin plus 3-MA. P, analyzed by ANOVA.

\section{Discussion}

The radiotherapy for thoracic malignant tumors may cause the RILI, one of the most common and severe complications. RILI usually starts 6 to 12 weeks after irradiation, as an exudative inflammation with the clinical features of interstitial pneumonia, and may proceed to a productive chronic inflammation lasting several months and terminating in scar formation, the lung fibrosis $(2,25)$. As a protease inhibitor, ulinastatin, a glycoprotein extracted from fresh human urine, inhibits the activity of proteolytic enzymes. Ulinastatin has been used in treatment of a variety of diseases, including acute pancreatitis, myocardial ischemia reperfusion injury and sepsis (26-28). In the present study, we establish a mouse model to investigate the possible effect of ulinastatin on the improvement of RILI, which is regarded as a new biologic treatment strategy.

Previous studies have indicated that ulinastatin has a protective effect on the lung injury $(3,29,30)$. In the pathogenesis of pulmonary diseases, including chronic obstructive pulmonary disease (COPD), acute lung injury and pulmonary hypertension, autophagy plays a dual role, with protective and injurious effects (31). Therefore, in this study, we have evaluated the protective effect of autophagy induced by ulinastatin on RILI. Electron microscopy has shown that ulinastatin keeps the structure intact of type I and II alveolar cells and increases the number of autophagosomes in the cytoplasm, which protects the lung tissue from radiation-induced damage. Observation by confocal microscope has also demonstrated that ulinastatin elevates the level of LC3, indicating the increase in the autophagy in RILI.

TGF- $\beta$ is a key cytokine and plays a role in antiinflammatory actions by controlling the homeostasis of extracellular matrix (32). In fibrotic organs, the expression levels of TGF- $\beta 1$ and its receptor were significantly increased. TGF- $\beta 1$ is a most important mediators to radioactive pulmonary fibrosis with the deposition of extracellular matrix $(33,34)$. Lionizing radiation may cause tissue hypoxia and generate a large amount of oxygen free radicals, which activate TGF- $\beta 1$ to mediate the differentiation of fibroblasts into myofibroblasts, the key effector in the process of fibrosis (35). Activation of myfibroblasts is needed $\alpha$-SMA (36) and type I collagen (Col1), including Col1A1 and Col1A2 (33). In addition, MMP-2 and MMP-9 play roles in the pathogenesis of pulmonary diseases (23). Therefore, TGF- $\beta 1$, Col1, $\alpha$-SMA, MMP-2 and MMP-9 are considered as indicators of pulmonary fibrosis. In our study, radiation increases the expression of all these genes. The ulinastatin treatment down-regulates Col1, $\alpha$-SMA, MMP-2 MMP-9, but upregulates TGF- $\beta 1$ expressions at the transcriptional and/ or translational levels. However, the administration of 3-MA, an autophagy inhibitor, alleviates the effects of ulinastatin, resulting in up-regulating $\alpha$-SMA and Col1, but down-regulating TGF- $\beta 1$, resulting in the destruction of type I and II alveolar cells and decrease in the number of autophagosomes. Interestingly, ulinastatin increase but 3-MA inhibits the expression of LC3. Our study has revealed the possible mechanism of ulinastatin protecting against the injury of the lung tissue in RILI through enhancement of autophagy and anti-inflammatory action with the increase in the levels of LC 3 and TGF- $\beta 1$, and reduction of pathogenesis of pulmonary diseases including fibroblasts with the decrease in the levels of $\alpha$-SMA, Col1A1, Col1A2, MMP-2 and MMP9.

\section{Conclusions}

In conclusion, ulinastatin protects the lung tissue against 
RILI in the mouse model. Ulinastatin up-regulates TGF- $\beta 1$ and LC3 that enhances autophagy, resulting in downregulating $\alpha$-SMA, Col1, MMP-2 and MMP9. Therefore, ulinastatin may be a useful medication to prevent from the lung fibrosis caused by radiation therapy for the thoracic cancer.

\section{Acknowledgments}

Funding: This study was supported by the Natural Science Foundation of Jilin Province (20160101170JC, 20200201541JC and 2019Q037). The Natural Science Foundation of Jilin Province provided the financial support.

\section{Footnote}

Reporting Checklist: The authors have completed the ARRIVE reporting checklist. Available at http://dx.doi. org/10.21037/tcr-19-3018

Data Sharing Statement: Available at http://dx.doi. org/10.21037/tcr-19-3018

Conflicts of Interest: All authors have completed the ICMJE uniform disclosure form (available at http://dx.doi. org/10.21037/tcr-19-3018). The authors have no conflicts of interest to declare.

Ethical Statement: The authors are accountable for all aspects of the work in ensuring that questions related to the accuracy or integrity of any part of the work are appropriately investigated and resolved. All animal tests were carried out in accordance with the US National Institute of Health (NIH) Guide for the Care and Use of Laboratory and approved by Institutional Animal Care and Use Committee (IACUC) and the ethics committee of the Jilin University (No. Jlzlyy-20180001).

Open Access Statement: This is an Open Access article distributed in accordance with the Creative Commons Attribution-NonCommercial-NoDerivs 4.0 International License (CC BY-NC-ND 4.0), which permits the noncommercial replication and distribution of the article with the strict proviso that no changes or edits are made and the original work is properly cited (including links to both the formal publication through the relevant DOI and the license). See: https://creativecommons.org/licenses/by-nc-nd/4.0/.

\section{References}

1. Santyr G, Fox M, Thind K, et al. Anatomical, functional and metabolic imaging of radiation-induced lung injury using hyperpolarized MRI. NMR Biomed 2014;27:1515-24.

2. Trott KR, Herrmann T, Kasper M. Target cells in radiation pneumopathy. Int J Radiat Oncol Biol Phys 2004;58:463-9.

3. Medhora M, Gao F, Wu Q, et al. Model development and use of ACE inhibitors for preclinical mitigation of radiation-induced injury to multiple organs. Radiat Res 2014;182:545-55.

4. Tahamtan R, Shabestani Monfared A, Tahamtani Y, et al. Radioprotective effect of melatonin on radiationinduced lung injury and lipid peroxidation in rats. Cell J 2015;17:111-20.

5. Wang J, Zhang YY, Cheng J, et al. Preventive and therapeutic effects of quercetin on experimental radiation induced lung injury in mice. Asian Pac J Cancer Prev 2015;16:2909-14.

6. Hu GD, Cai SX, Chen B, et al. Effects of serum of the rats ventilated with high tidal volume on endothelial cell permeability and therapeutic effects of ulinastatin. Chin Med J (Engl) 2006;119:1374-80.

7. Inoue $\mathrm{K}$, Takano $\mathrm{H}$, Yanagisawa $\mathrm{R}$, et al. Protective role of urinary trypsin inhibitor in acute lung injury induced by lipopolysaccharide. Exp Biol Med (Maywood) 2005;230:281-7.

8. Katoh $\mathrm{H}$, Ishikawa $\mathrm{H}$, Hasegawa $\mathrm{M}$, et al. Protective effect of urinary trypsin inhibitor on the development of radiation-induced lung fibrosis in mice. J Radiat Res 2010;51:325-32.

9. Bao P, Gao W, Li S, et al. Effect of pretreatment with high-dose ulinastatin in preventing radiationinduced pulmonary injury in rats. Eur J Pharmacol 2009;603:114-9.

10. Bao P, Zhao W, Li Y, et al. Protective effect of ulinastatin in patients with non-small cell lung cancer after radiation therapy: a randomized, placebo-controlled study. Med Oncol 2015;32:405.

11. Cao C, Yin C, Shou S, et al. Ulinastatin Protects Against LPS-Induced Acute Lung Injury By Attenuating TLR4/NF-kappaB Pathway Activation and Reducing Inflammatory Mediators. Shock 2018;50:595-605.

12. Song B, Bian Q, Shao CH, et al. Ulinastatin reduces the resistance of liver cancer cells to epirubicin by inhibiting 
autophagy. PLoS One 2015;10:e0120694.

13. Li HF, Zhao SX, Xing BP, et al. Ulinastatin suppresses endoplasmic reticulum stress and apoptosis in the hippocampus of rats with acute paraquat poisoning. Neural Regen Res 2015;10:467-72.

14. Vilgelm AE, Pawlikowski JS, Liu Y, et al. Mdm2 and aurora kinase a inhibitors synergize to block melanoma growth by driving apoptosis and immune clearance of tumor cells. Cancer Res 2015;75:181-93.

15. Matsuzawa-Ishimoto Y, Hwang S, Cadwell K. Autophagy and Inflammation. Annu Rev Immunol 2018;36:73-101.

16. Netea-Maier RT, Plantinga TS, van de Veerdonk FL, et al. Modulation of inflammation by autophagy: Consequences for human disease. Autophagy 2016;12:245-60.

17. McDonald S, Rubin P, Phillips TL, et al. Injury to the lung from cancer therapy: clinical syndromes, measurable endpoints, and potential scoring systems. Int J Radiat Oncol Biol Phys 1995;31:1187-203.

18. Mi Y, Wang W, Zhang C, et al. Autophagic Degradation of Collagen 1A1 by Cortisol in Human Amnion Fibroblasts. Endocrinology 2017;158:1005-14.

19. Wang G, Liu Y, Zhou SF, et al. Effect of Somatostatin, Ulinastatin and Gabexate on the Treatment of Severe Acute Pancreatitis. Am J Med Sci 2016;351:506-12.

20. Vescarelli E, Pilloni A, Dominici F, et al. Autophagy activation is required for myofibroblast differentiation during healing of oral mucosa. J Clin Periodontol 2017;44:1039-50.

21. Koesters R, Kaissling B, Lehir M, et al. Tubular overexpression of transforming growth factor-beta 1 induces autophagy and fibrosis but not mesenchymal transition of renal epithelial cells. Am J Pathol 2010;177:632-43.

22. Xiao J, Zhu X, Ji G, et al. Ulinastatin protects cardiomyocytes against ischemiareperfusion injury by regulating autophagy through mTOR activation. Mol Med Rep 2014;10:1949-53.

23. Yang B, Gao M, Wang K, et al. Intraintestinal administration of ulinastatin protects against sepsis by relieving intestinal damage. J Surg Res 2017;211:70-8.

24. Li WD, Li NP, Song DD, et al. Metformin inhibits endothelial progenitor cell migration by decreasing matrix metalloproteinases, MMP-2 and MMP-9, via the AMPK/mTOR/autophagy pathway. Int J Mol Med 2017;39:1262-8.

25. Wang DJ, Liu JX, Yin BL. Protective effects of ulinastatin on the lung injury during cardiopulmonary bypass. Zhong Nan Da Xue Xue Bao Yi Xue Ban 2005;30:670-2.

26. Leng YX, Yang SG, Song YH, et al. Ulinastatin for acute lung injury and acute respiratory distress syndrome: A systematic review and meta-analysis. World J Crit Care Med 2014;3:34-41.

27. Martin M, Lefaix J, Delanian S. TGF-beta1 and radiation fibrosis: a master switch and a specific therapeutic target? Int J Radiat Oncol Biol Phys 2000;47:277-90.

28. Mizumura K, Cloonan SM, Haspel JA, et al. The emerging importance of autophagy in pulmonary diseases. Chest 2012;142:1289-99.

29. Fu MY, He YJ, Lv X, et al. Transforming growth factorbeta1 reduces apoptosis via autophagy activation in hepatic stellate cells. Mol Med Rep 2014;10:1282-8.

30. Kim SI, Na HJ, Ding Y, et al. Autophagy promotes intracellular degradation of type I collagen induced by transforming growth factor (TGF)-beta1. J Biol Chem 2012;287:11677-88.

31. Rodt T, von Falck C, Dettmer S, et al. Micro-computed tomography of pulmonary fibrosis in mice induced by adenoviral gene transfer of biologically active transforming growth factor-beta1. Respir Res 2010;11:181.

32. Falanga V, Zhou L, Yufit T. Low oxygen tension stimulates collagen synthesis and COL1A1 transcription through the action of TGF-beta1. J Cell Physiol 2002;191:42-50.

33. Henriksen NA. Systemic and local collagen turnover in hernia patients. Dan Med J 2016;63:B5265.

34. Ju W, Zhihong Y, Zhiyou Z, et al. Inhibition of alpha-SMA by the ectodomain of FGFR2c attenuates lung fibrosis. Mol Med 2012;18:992-1002.

35. Ning XH, Ge XF, Cui Y, et al. Ulinastatin inhibits unilateral ureteral obstruction-induced renal interstitial fibrosis in rats via transforming growth factor beta (TGFbeta)/Smad signalling pathways. Int Immunopharmacol 2013;15:406-13.

36. Li D, Ji H, Zhao B, et al. Therapeutic Effect of Ulinastatin on Pulmonary Fibrosis via Downregulation of TGF- $\beta 1$, TNF- $\alpha$ and NF-кB. Mol Med Rep 2018;17:1717-23.

Cite this article as: Zhang G, Du Y, Sun N, Sun Y, Zhang L, Li X, Li X. Ulinastatin enhances autophagy against radiationinduced lung injury in mice. Transl Cancer Res 2020;9(7):41624172. doi: 10.21037/tcr-19-3018 